\title{
Surveillance et confortement des fondations d'ouvrages d'art en site aquatique Aspect hydraulique
}

\author{
Surveillance and reinforcement \\ of foundations for structure in aquatic sites \\ The hydraulic aspect
}

Marcel Ramette

Ingénieur Chargé de Mission au «Laboratoire

National d'Hydraulique » Chatou.

Conférence faite à Tours au cours des journées organisées par l'Ecole Nationale des Ponts et Chaussées (formation permanente) en Novembre 1980.

J'ai partagé mon exposé en trois thèmes principaux, car il m'a semblé nécessaire en premier lieu de décrire les phénomènes qui se produisent dans une rivière naturelle, c'est-à-dire non perturbée par des ouvrages quelconques.

Pour guérir un malade, si maladie il y a, encore faut-il savoir comment fonctionne son organisme, celà me paraît essentiel.

En second lieu et à la lumière du premier thème, nous examinerons la façon dont réagit cette rivière naturelle quand on perturbe son cours par l'édification d'ouvrages, que ce soit un pont, un endiguement, un seuil ou toute autre construction artificielle, et cette réaction nous permettra de définir un nouvel état d'équilibre de la rivière, dont bien entendu le projeteur de l'ouvrage est supposé avoir tenu compte, pour que la stabilité de cet ouvrage soit assurée au moins pendant les premières années de son existence.

Et enfin, puisque le thème essentiel de ces journées est le confortement des fondations des ouvrages, nous supposerons que des "agressions" non prévues à l'époque de l'élaboration du projet viennent perturber l'équilibre de la rivière et mettent en péril la stabilité des ouvrages.

Nous passerons en revue ces "agressions" et sans empiéter sur les sujets des autres conférenciers nous tenterons de définir les grandes lignes des moyens de défense pour conforter, sans aggraver davantage.

\section{La rivière non perturbée}

Une rivière est le résultat d'un équilibre complexe et très précaire entre les caractéristiques du débit liquide qu'elle doit écouler et les caractéristiques des sédiments et de la vallée dans laquelle elle creuse son lit.

Cet équilibre se traduit

- d'une part, en plan par une forme du lit, qui est caractérisé, soit par un aspect rectiligne, soit par des méandres.

Pour compliquer les phénomènes, ces méandres glissent bien souvent vers l'aval et on peut déjà concevoir que ces glissements, s'ils ne sont pas fixés, vont constituer une forme d'agression sur laquelle nous reviendrons tout à l'heure.

- d'autre part, le lit se caractérise par des sections verticales, désignées par "profils en travers", qui constituent généralement deux lits distincts : un lit mineur qui évacue à limite de débordement le débit maximal le plus fréquent $Q_{a}$, disons la crue annuelle, et un lit majeur qui évacue l'excédent des crues les plus importantes.

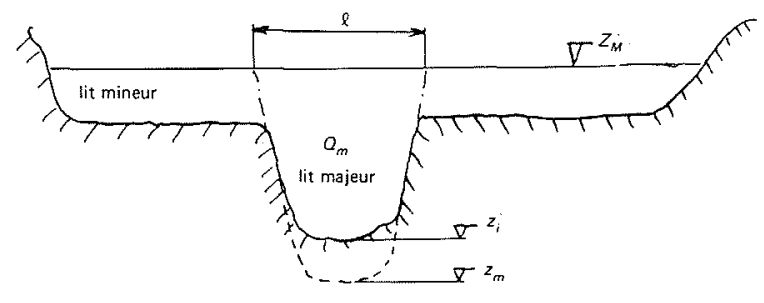

Figure 1

Le débit du lit mineur s'écoule en général sur des alluvions mobiles et peut donc se déformer au cours des crues. Par contre, le lit majeur, constitué en général de plantations, de zones urbanisées peut être considéré comme inamovible.

La déformation du lit mineur est évidemment très importante à connaître pour les problèmes qui nous intéressent.

Comment se déforme-t-il ?

A partir d'un certain seuil de débit, les sédiments se mettent en mouvement et leur déplacement peut s'effectuer selon différents modes qui sont grosso-modo les suivants : 
- des rides ou des dunes si $d>1 \mathrm{~mm}$ (charriage)

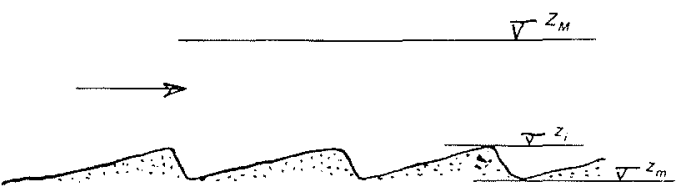

Figure 2

- une saltation plus ou moins généralisée si $d<1 \mathrm{~mm}$ (suspension)

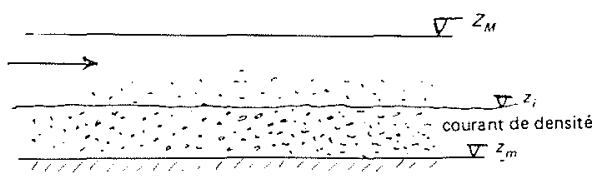

Figure 3

Dans l'un et l'autre cas, il semble bien que l'essentiel du transport solide se fasse dans une couche $z_{i}-z_{m}$ : $z_{i}$ étant la cote moyenne initiale du fond ; $z_{m}$ la cote moyenne des fond non perturbés.

Dans le premier cas, $z_{i}$ et $z_{m}$ seraient respectivement la cote de crête et la cote de pied des dunes ou rides et dans le second cas, $\left(z_{i}-z_{m}\right)$ serait l'épaisseur du courant de densité.

Si $z_{i}$ est connu, $z_{m}$ peut être évalué par la relation expérimentale suivante :

$$
z_{m}=Z_{M}-0,73 \frac{q^{2 / 3}}{d_{m}^{1 / 6}}
$$

dans laquelle : (fig. 4)

- $Z_{M}$ est la cote du plan d'eau atteinte par la crue de débit $Q$ et que l'on suppose connue ;

$-d_{m}$ est le diamètre moyen des sédiments (en $m$ ); $-q=Q_{m} / l$, est le débit $Q_{m}$ par unité de largeur $l \mathrm{du}$ lit mineur

$Q_{m}$ est différent et plus grand que $Q_{a}$ puisque $Q_{a}$ est le débit à limite de débordement.

On pourra estimer $Q_{m}$ par la relation de ManningStrickler :

$$
Q_{m}=32 \cdot \frac{S^{5 / 3}}{p^{2 / 3}} \cdot J^{1 / 2}
$$

dans laquelle

32 est une valeur moyenne du coefficient de rugosité,

$S$ : section mouillée initiale sous la cote $Z_{M}$,

$p$ : périmètre mouillé initial sour la cote $Z_{M}$,

$J$ : pente de la ligne d'eau.

On s'assurera évidemment que $Q_{m}$ ainsi calculé a une valeur telle que :

$$
Q_{a}<Q_{m}<Q
$$

On retiendra que $z_{m}$ correspond à la cote des fonds en-dessous de laquelle les sédiments n'ont pas été déplacés.

L'épaisseur des fonds en mouvement $\left(z_{i}-z_{m}\right)$ peut être considérable. C'est ainsi que sur certains cours d'eau coulant sur des sédiments fins, $\left(z_{i}-z_{m}\right)$ peut atteindre $10 \mathrm{~m}$ en période de crue.
Sur le Tigre à Bagdad avec $0,1<d_{m}<0,2 \mathrm{~mm}$ on a mesuré une épaisseur $\left(z_{i}-z_{m}\right)$ de sédiments en mouvement de $7 \mathrm{~m}$ sur une largeur de $250 \mathrm{~m}$ au cours d'une crue de $2000 \mathrm{~m}^{3} / \mathrm{s}$. Après la crue, les fonds avaient repris leur profil initial laissant l'illusion que rien d'important ne s'était passé.

Nous donnons ci-après les caractéristiques schématiques du lit du Tigre observé pour $Q=2000 \mathrm{~m}^{3} / \mathrm{s}$.

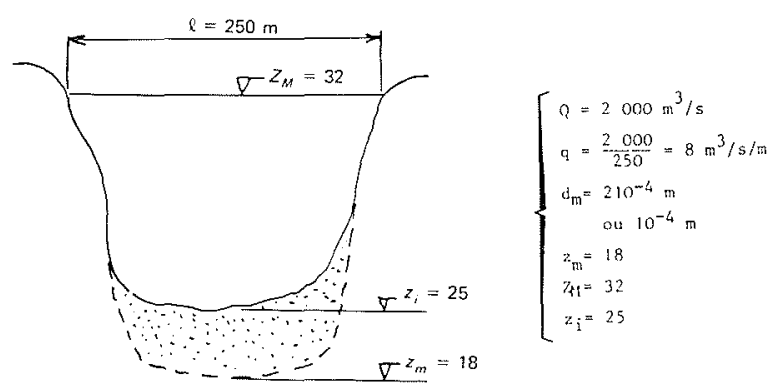

Figure 4

L'application de la formule (1) donne :

$$
z_{m}=32-0,73 \frac{8^{2 / 3}}{\left(d_{m}\right)^{1 / 6}}
$$

soit $z_{m}=19,9 \mathrm{~m}$ pour $d_{m}=2.10^{-4} \mathrm{~m}$

ou $\quad z_{m}=18,4 \mathrm{~m}$ pour $d_{m}=10^{-4} \mathrm{~m}$

au lieu des 18 mètres mesurés.

On pourrait citer d'autres exemples montrant que la relation (1) donne un ordre de grandeur de $z_{m}$ assez proche de la réalité.

\section{Influence de l'implantation d'ouvrages sur la rivière}

\section{Premier cas : Rivière stable en plan}

Reprenons la figure 1 et supposons un pont franchissant le lit mineur, avec largeur entre culées $l=l_{1}$ et route d'accès insubmersible à la crue de débit $Q$. (fig. 5)

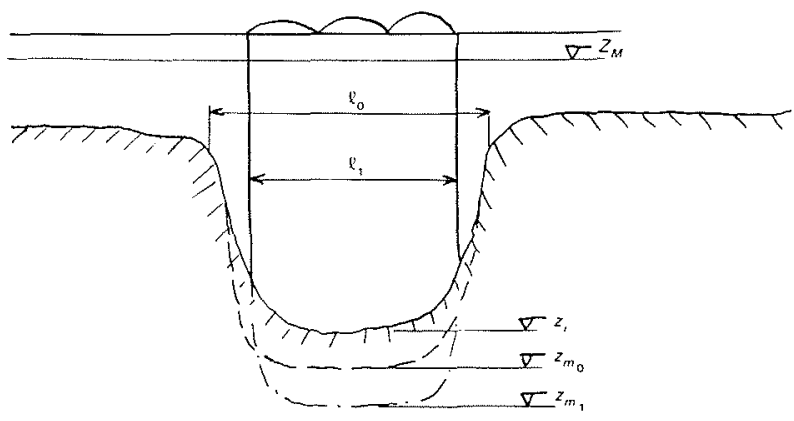

Figure 5

Tout le débit est donc concentré sur la largeur $l_{1}$. Par hypothèse la rivière est stable en plan et contenue par des endiguements par exemple; il n'y a donc pas de risques d'érosion des berges, ni de contournement de l'ouvrage, ni de variation d'angle d'attaque du courant. 


\section{$l^{\grave{e} r e}$ perturbation (fig. 6)}

Elle est d'ordre hydraulique : le pincement du lit provoque un remous d'exhaussement $\Delta Z$ en amont du pont, mais la cote de l'eau en aval reste sensiblement identique à ce qu'elle était avant la construction du pont : $Z=Z_{M}$.

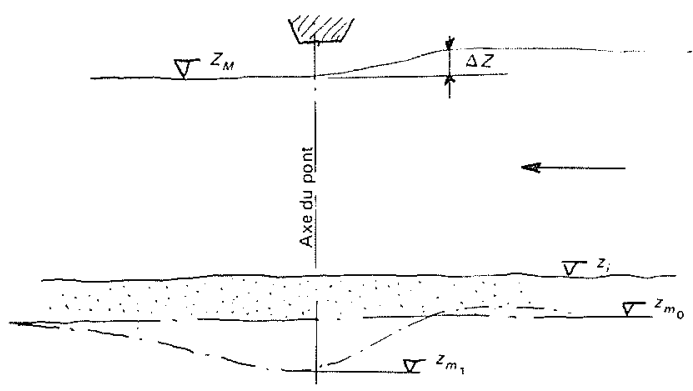

Figure 6

\section{$2^{e}$ perturbation}

En cours de crue, dans la situation naturelle, les fonds étaient mobilisés depuis la cote $z_{i}$ jusqu'à la cote $z_{m o}$

$\left(\begin{array}{lll}l_{m} & : \text { dargeur initiale du lit mineur. }\end{array}\right.$

Après la construction du pont, la nouvelle cote $z_{m_{1}}$ est la suivante :

$$
z_{m_{1}}=Z_{M}-0,73 \frac{q_{1}^{2 / 3}}{d_{m}^{1 / 6}}
$$

avec $\left\{\begin{array}{l}q_{1}=\frac{Q}{l_{1}}>q_{0} \\ Q: \text { débit total entre culées, } \\ l_{1}: \text { largeur entre culées. }\end{array}\right.$

Il y a donc, uniquement du fait de la concentration du courant due à la présence des culées, un abaissement de la cote des fonds non perturbés dont la valeur est la suivante :

$$
z_{m_{0}}-z_{m_{1}}=\frac{0,73}{d_{m}^{1 / 6}}\left[q_{1}^{2 / 3}-q_{0}^{2 / 3}\right.
$$

\section{$3^{e}$ perturbation}

Elle est due à la présence des piles qui localement vont engendrer un affouillement sous la cote $z_{m_{1}}$.

Sans entrer dans le détail du phénomène complexe de cet affouillement, disons qu'un ordre de grandeur du surcreusement maximal créé en tête de pile est de $2 D$ environ, si $D$ est le maître couple de la pile compté perpendiculairement à la direction du courant (Fig. 7).
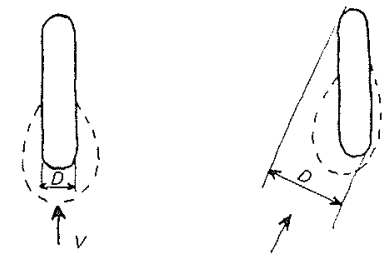

Figure 7

On conçoit l'importance de la direction du courant sur l'affouillement créé par une pile allongée.

En résumé, on retiendra qu'au pied d'une pile, les fonds d'une rivière ne sont pas mobilisés sous la cote moyenne :

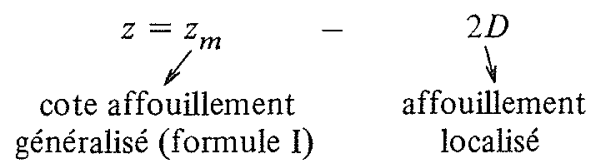

\section{Deuxième cas - Rivière instable en plan}

La construction d'un pont et de ses accès sur un tel lit contribue évidemment à fixer localement la rivière, du moins temporairement, au droit du franchissement, mais l'instabilité existe toujours en amont et en aval; il en résulte un lit "torturé" qui essaie de retrouver sa liberté de déplacement, ce qui se traduit toujours par des angles d'attaque des piles très variables dans le temps et parfois par un court-circuitage pur et simple de l'ouvrage.

Beaucoup d'ouvrages ont péri du fait d'une attaque des piles sous un angle avoisinant $90^{\circ}$. Le pont de Moundou au Tchad en est un exemple.

Certains se sont retrouvés isolés, "ignorés", par le cours d'eau qui au travers du remblai d'accès s'était creusé un nouveau lit.

Dans les deux cas cités, il s'agit de l'influence des ouvrages sur la rivière; le projeteur est censé ne pas ignorer les phénomènes décrits sous peine d'être amené à envisager des travaux de confortement dès les premières années de la vie de l'ouvrage.

Il est censé savoir, en particulier, qu'une implantation d'ouvrage sur une rivière instable nécessite des travaux de fixation du lit qui vont de pair avec la construction de l'ouvrage proprement dit et que, de plus, les piles de l'ouvrage auront intérêt à être constituées d'un ensemble de fûts cylindriques, plutôt que d'un voile continu, pour minimiser l'effet d'une incidence plus ou moins grande du courant.

Nous nous placerons, dans ce qui suit, dans le cas d'ouvrages anciens et qui du fait d'un certain nombre de travaux entrepris sur la rivière, que nous énumérerons, voient leur stabilité menacée.

\section{Influence de travaux en rivière sur la stabilité des ouvrages}

Il faut bien se persuader que tous les travaux sur une rivière peuvent affecter, à plus ou moins long terme, l'équilibre de cette rivière même en un point éloigné du lieu de ces travaux et donc compromettre la stabilités des ouvrages qui y sont implantés. 


\section{Augmentation du débit (Fig. 8)}

A titre d'exemple nous citerons des travaux d'endiguements locaux réalisés en vue de retarder le débordement d'une rivière.

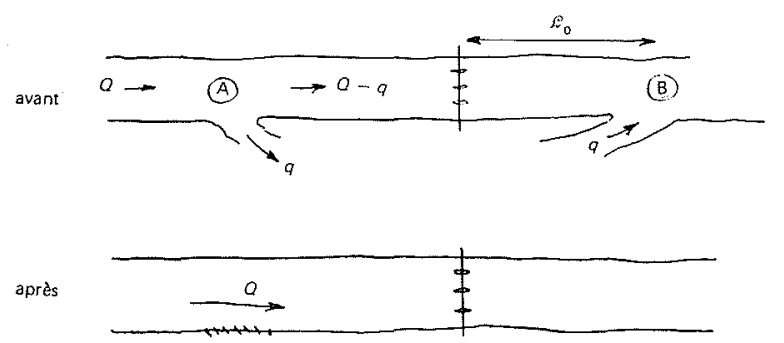

Figure 8

Le débit au droit de l'ouvrage qui était initialement $(Q-q)$ augmente de la valeur $q$ quand l'endiguement est réalisé; c'est une première conséquence évidente qui a comme corollaire une augmentation des vitesses en aval du point $A$.

Le profil en long des fonds présentait l'aspect suivant (Fig. 9) : une certaine pente $J_{0}$ en amont de $A$, une pente $J_{1}$ plus forte en aval, simplement parce que le lit s'était organisé pour écouler le même débit solide en aval de $A$ bien que le débit liquide soit plus faible.

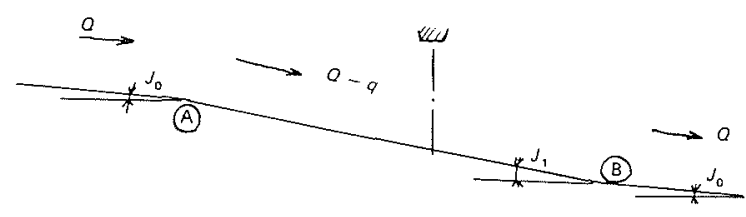

Figure 9

Si $B$ était approximativement le point de restitution du débit $q$ de débordement, le lit retrouvait en aval de $B$ sa pente initiale $J_{0}$.

Dès que l'endiguement est réalisé, le débit liquide devient uniforme $=Q$ et le profil en long des fonds tend lui aussi vers la pente uniforme $J_{0}$ pour écouler le même débit solide.

Le profil en long des fonds présente alors l'aspect suivant (Fig. 10) :

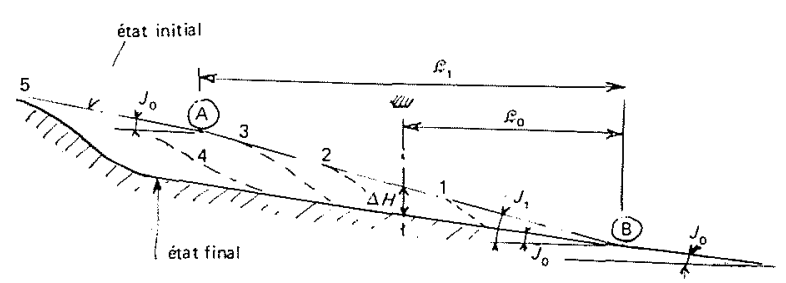

Figure 10

- Au droit de l'ouvrage les fonds s'abaissent donc de :

$$
\Delta H_{\max }=\left(J_{1}-J_{0}\right) \mathcal{E}_{0}
$$

abaissement qui peut être très important selon la valeur de $\left(J_{1}-J_{0}\right)$ et de $\mathscr{S}_{0}$ et qui, bien entendu, peut compromettre la stabilité des fondations.
- On notera que l'abaissement maximum se situe au droit de $A$ et a pour équation :

$$
\Delta H_{\max }=\left(J_{1}-J_{0}\right) \mathfrak{L}_{1}
$$

Cet abaissement qui intéresse tous les ouvrages en amont de $B$ ne se fait évidemment pas instantanément, il peut s'étaler sur de nombreuses années; il est fonction de l'importance des crues, de leur durée, de la granulométrie, mais il est inexorable si un point dur quelconque, naturel ou artificiel, ne vient en stopper le processus qui est un processus d'érosion régressive.

C'est le phénomène qui s'est produit pour le lit du Gapeau, rivière côtière se jetant en Méditerranée près d'Hyères.

On notera qu'avant que le processus d'érosion régressive n'ait eu le temps de se développer et d'atteindre l'ouvrage, il y a de toutes façons un abaissement de la cote des fonds non perturbés $z_{m}$, puisque la relation (I) est à utiliser avec une valeur de $q$ calculée non plus avec $(Q-q)$ mais avec $Q$ dans la région $(A-B)$.

\section{Diminution de la cote de l'eau en aval de l'ouvrage}

Il y a beaucoup de travaux en rivière qui se traduisent par un abaissement du plan d'eau, à débit égal, au droit d'un ouvrage, et par conséquent par une augmentation locale des vitesses; parmi ces travaux nous en citerons trois.

\section{a) Recalibrage du lit de part et d'autre d'un pont}

Des travaux de recalibrage de rivière sont entrepris en général pour augmenter la capacité d'écoulement du lit mineur ; c'est-à-dire qu'à cote égale le débit soit plus élevé ou bien encore, qu'à un même débit corresponde après aménagement une cote plus basse.

La figure 11 représente cette rivière avant travaux, et la figure 12 , cette même rivière après travaux.
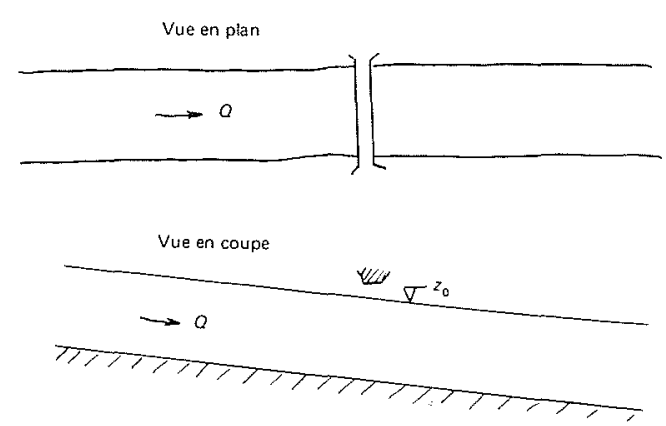

Figure 11

A débit égal (fig. 12b)

- l'application de la formule (I) montre que $z_{m}$ s'abaisse de $\left(Z_{0}-Z_{1}\right)$;

- que l'abaissement de cote de $Z_{0}$ à $Z_{1}$ se traduit par une augmentation des vitesses qui peuvent devenir suffisantes pour entrainer les protections existantes. 

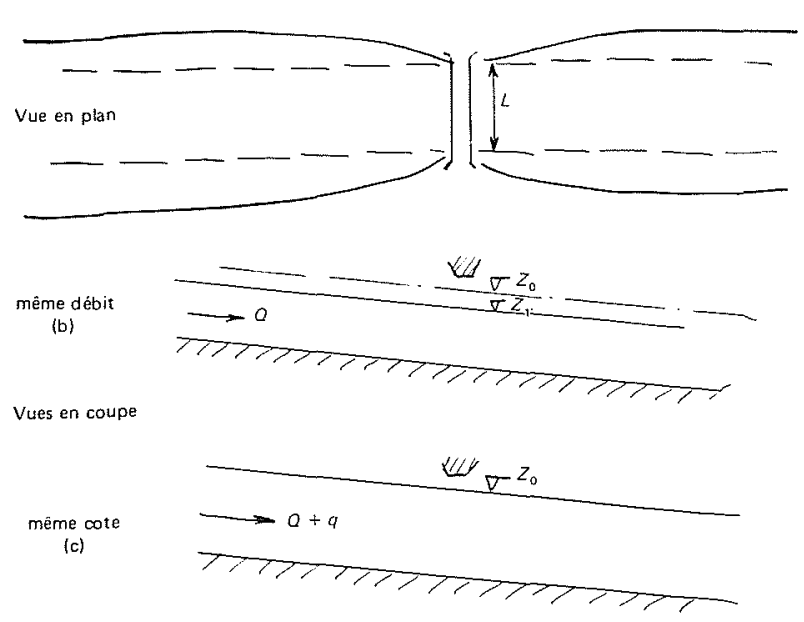

Figure 12

\section{A cotes égales (fig. $12 \mathrm{c}$ )}

- l'application de la formule (I) montre que $z_{m}$ s'abaisse de

$$
\frac{0,73}{d_{m}^{1 / 6}}\left[\frac{(Q+q)^{2 / 3}-Q^{2 / 3}}{L^{2 / 3}}\right]
$$

- que les vitesses augmentent dans le rapport $\frac{Q+q}{Q}$

Dans l'un et l'autre cas, il y a une aggravation de la profondeur d'affouillement et une augmentation des vitesses qui peuvent se traduire par la ruine de l'ouvrage.

C'est ainsi qu'après la réalisation du recalibrage du lit de l'Agly (Pyrénées Orientales), le vieux pont qui permettait le franchissement de la route $C D 11$ a été emporté dès la première crue.

\section{b) Coupures de méandres}

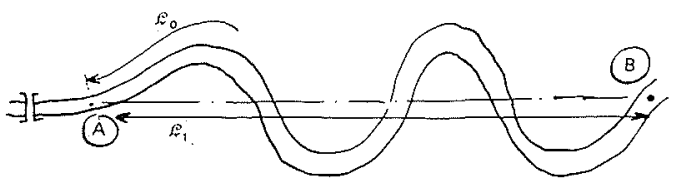

Figure 13

Imaginons par exemple une coupure de méandres en aval d'un pont entre deux points $A$ et $B$. (fig. 13)

Le lit initial avait une longueur $\mathcal{E}_{0}$; le lit rectifié a une longueur $\mathscr{L}_{1} \cdot\left(\mathscr{P}_{0}>\mathfrak{P}_{1}\right)$.

$\mathrm{Ce}$ lit rectifié, dès sa réalisation, a une pente plus forte $\left(J_{1}\right)$ que le lit initial $\left(J_{0}\right)$. En effet en désignant par $Z_{A}$ et $Z_{B}$ l'altitude des fonds aux points $A$ et $B$, on a :

et

$$
\begin{gathered}
J_{0}=\frac{Z_{A}-Z_{B}}{\mathscr{P}_{0}} \\
J_{1}=J_{0} \frac{\mathscr{f}_{0}}{\mathscr{E}_{1}}
\end{gathered} \Rightarrow J_{1}>J_{0}
$$

Cette pente $J_{1}$ ne peut se maintenir car $J_{0}$ est la pente d'équilibre permettant d'écouler le débit liquide et le débit solide.

Le lit rectifié va donc basculer autour du point $B$ pour retrouver la pente $J_{0}$ (Fig. 14).

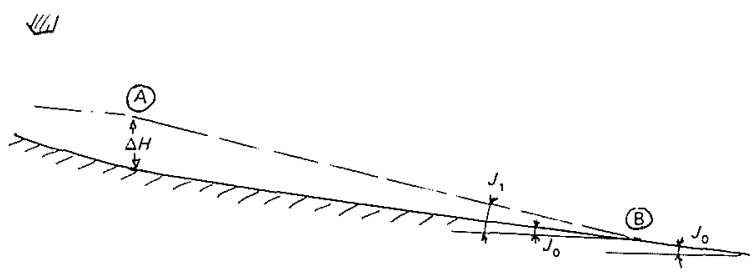

Figure 14

En $A$, les fonds vont s'abaisser de :

$$
\Delta H=\left(J_{1}-J_{0}\right) \mathfrak{P}_{1}=J_{0}\left(\mathfrak{e}_{0}-\mathfrak{P}_{1}\right)
$$

et bien entendu une érosion régressive se développera vers l'amont, c'est-à-dire vers le pont.

\section{c) Dragages en aval d'un pont}

Les dragages en aval d'un pont ont toujours une influence néfaste, amis qui est plus ou moins sérieuse, la longueur de dragage et sa profondeur affectant sensiblement ou peu la cote de l'eau en aval de l'ouvrage.

Imaginons par exemple, un dragage sur toute la largeur de la rivière mais sur une longueur assez faible, 100 mètres par exemple (Fig. 15).

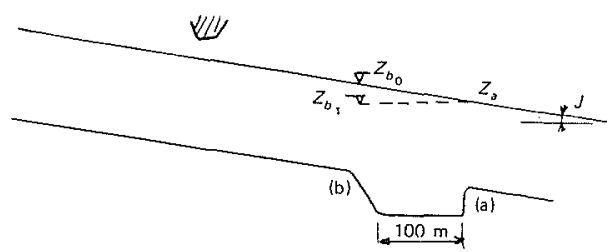

Figure 15

La cote de l'eau en aval du dragage reste égale à ce qu'elle était : $z_{a}$; au pis aller, si le dragage est profond, le plan d'eau est sensiblement horizontal et la différence entre la cote initiale $z_{b_{0}}$ en amont de dragage et $z_{p_{1}}$ après dragage est :

$$
z_{b_{0}} \quad z b_{1}=100 J
$$

et en imaginant une pente forte $J=10^{-3}$ :

$$
z_{b_{0}}-z_{b_{1}}=0,1 \mathrm{~m}
$$

Un abaissement aussi faible de l'eau ne peut avoir que des conséquences faibles sur l'équilibre des fonds, la souille de dragage se comblera progressivement sans pratiquement affecter la cote des fonds au droit de l'ouvrage (Fig. 16).

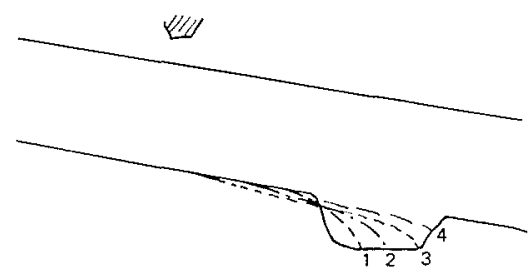

Figure 16 
Il n'en va pas du tout de même si les dragages affectent une longueur importante du cours d'eau.

$\mathrm{Si}$ au lieu des 100 mètres envisagés il s'agit d'un kilomètre de dragage, la dénivelée $Z_{0}-z_{1}$ atteint 1 mètre et peut évidemment avoir des conséquences facheuses pour l'ouvrage du fait de l'érosion régressive qui se développe vers l'amont (Fig. 17); et encore nous plaçons-nous dans le cas favorable où nous supposons que la cote du plan d'eau $z_{a}$ en aval du dragage reste constante alors qu'en fait elle ne peut que s'abaisser si aucun point dur ne maintient les fonds en aval de la souille de dragage.

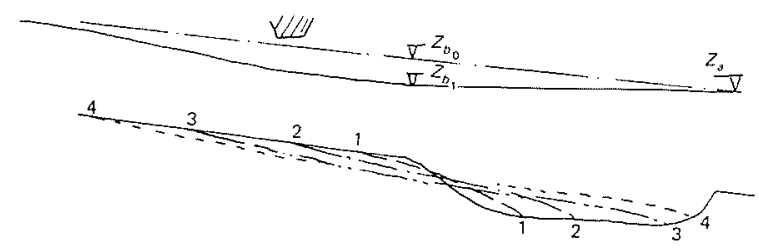

Figure 17

\section{Dragages en amont d'un ouvrage}

Supposons que $G_{\mathrm{m}^{3}}$ soit l'apport solide moyen annuel d'une rivière et que par dragage on en prélève $g_{\mathrm{m} 3}$ en amont d'un certain ouvrage.

La rivière qui s'était adaptée pour écouler le débit solide $G_{\mathrm{m}} 3$ doit maintenant s'adapter pour écouler un débit solide moindre $(G-g) \mathrm{m}^{3}$ et doit donc changer ses caractéristiques géométriques.

Si les berges sont fixées, le seul paramètre géométrique qui puisse varier est la pente du lit et on conçoit que cette pente ne puisse que diminuer d'une valeur initiale $J_{0}$ à une valeur finale $J_{1}$ correspondant au nouvel équilibre.

$J_{1}$ est aisément calculable à partir des équations de Meyer-Peter :

$$
G_{\mathrm{m}^{3} / \mathrm{s}}=K L\left[H J-\tau_{c}\right]^{3 / 2}
$$

et de Manning-Strickler :

$$
Q=k L H^{5 / 3} J^{1 / 2}
$$

dans lesquelles

$$
\left\{\begin{array}{l}
K \# 20 \\
L \text { est la largeur de la rivière } \\
H \text { sa profondeur } \\
J \text { la pente } \\
\tau_{c}=0,08 d_{m y} \\
k: \text { coefficient de Manning-Strickler } \# 32 .
\end{array}\right.
$$

On trouvera aisément tous calculs faits

$$
J_{1}=\left[\frac{\left(\frac{G-g}{G}\right)^{2 / 3}\left[\left(\frac{Q}{k L}\right)^{3 / 5} J_{0}^{7 / 10}-\tau_{c}\right]+\tau_{c}}{\left(\frac{Q}{k L}\right)^{3 / 5}}\right]^{10 / 7}
$$

A la limite, si tout l'apport solide est prélevé, on trouvera (avec $g=G$ ):

$$
J_{1}=\tau_{c}^{10 / 7} \cdot\left(\frac{k L}{Q}\right)^{30 / 35}
$$

ou encore en explicitant $\tau_{c}$, avec $k \# 32$ et $q=\frac{Q}{L}$. on obtient :

$$
J_{1}=0,53 d_{m}^{1 / 43} q^{-0,86}
$$

C'est là l'expression de la pente d'équilibre que prend une rivière lorsque tout apport solide est bloqué. $J_{1}$ est donc calculable et on retiendra qu'il est toujours inférieur à $J_{0}$.

Dans ces conditions, comment évoluent les fonds à l'aval d'une zone de dragage $A$ en faisant l'hypothèse, optimiste, d'un point dur aval $B$ fixant les fonds? On peut le schématiser à l'équilibre par la figure 18 :

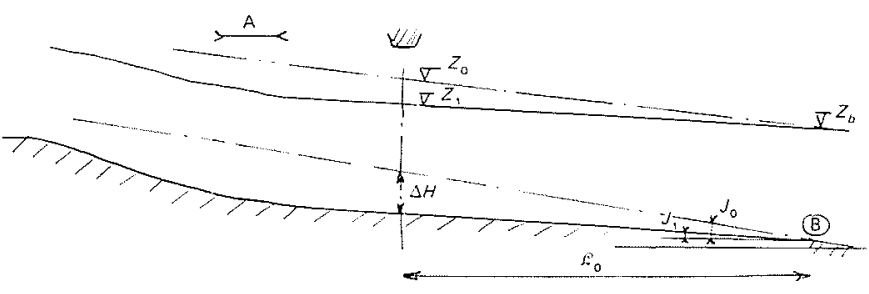

Figure 18

L'abaissement des fonds au droit de l'ouvrage est donné par l'équation :

$$
\Delta H=\left(J_{0}-J_{1}\right) \mathscr{P}_{0}
$$

Il est d'autant plus important que $J_{1}$ est petit, c'està-dire que $g$ est important vis-à-vis de $G$; il est d'autre par directement proportionnel à $\mathscr{P}_{0}$, et $\mathscr{f}_{0}$, si aucun point dur ne vient bloquer l'érosion, peut très bien être la distance de l'ouvrage à la mer !

$\Delta H$ peut donc être considérable et on conçoit que l'influence de dragages intensifs puisse être catastrophique sur la stabilité d'ouvrages, qu'ils soient en aval

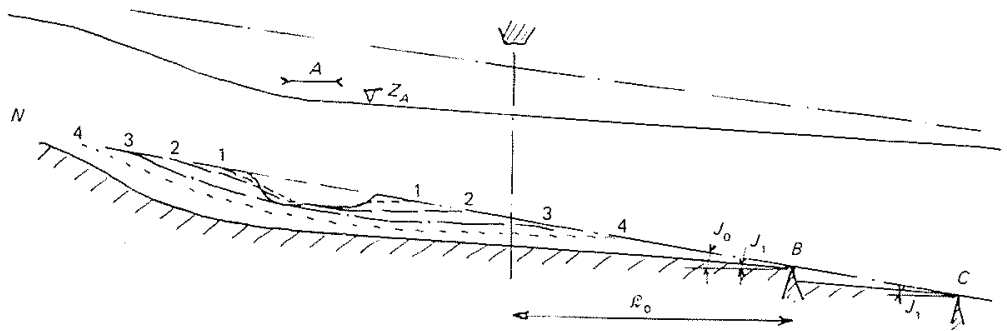

Figure 19 
des dragages comme nous venons de le voir, ou en amont comme nous l'avons envisagé dans l'exemple précédent puisque l'influence de l'érosion régressive est dans ce cas renforcée par l'abaissement corrélatif du plan d'eau en aval de la zone de dragage.

Là encore, la situation d'équilibre telle que nous l'avons représentée sur la figure 18 n'est pas instantanée et peut demander des années d'évolution; la progression du profil en long des fonds pour tendre vers cette situation d'équilibre peut se schématiser comme suit en supposant des points fixes en $B$ et $C$ (Fig. 19).

Il est théoriquement possible de calculer la cote $Z_{A}$ du plan d'eau au droit des dragages $A$ à partir des conditions aux limites aval et des différentes pentes $J_{1}<J_{0}$ que le cours d'eau rencontre; il y a bien évidemment en amont de $A$ un remous d'abaissement par rapport à la situation naturelle, remous d'abaissement qui s'éteint quelque part en $N$, limite de la perturbation provoquée par le dragage.

Si les berges sont mal ou peu protégées en aval de la zone de dragage, le cours d'eau au lieu de diminuer sa pente par emprunt de matériaux sur le fond aura tendance à diminuer sa pente en augmentant la longueur de son cours c'est-à-dire à éroder ses berges pour former des méandres (Fig. 20).

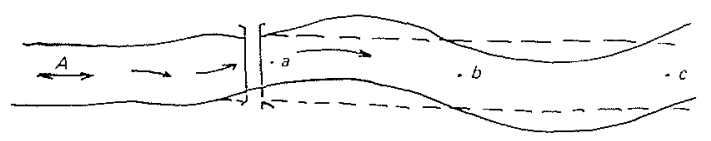

Figure 20

C'est en effet pour lui le moyen le plus rapide et, on peut le démontrer, le plus économique sur le plan énergétique, pour tendre vers la pente d'équilibre $J_{1}$

On peut concevoir le type d'inconvénients qui se présente pour les ouvrages en aval de $A$ dont les moindres sont:

- soit des attaques localisées des berges avec amorce de développement de méandres ;

- soit des risques d'attaque des piles sous une incidence plus ou moins grande.

A l'actif d'un tel processus, on peut dire que les cotes du fond aux différents nœuds du méandre $a, b, c$ varient peu par rapport à la situation naturelle, puisque le méandre par augmentation de la longueur du cours d'eau entre $a, b, c$ permet théoriquement de diminuer la pente sans toucher à l'altitude de ces points :

$$
\frac{J_{1}}{J_{0}}=\frac{\overline{a b}}{\overparen{a b}}=\frac{\overline{b c}}{\overparen{b c}}=\ldots
$$

En fait, la situation décrite est toute théorique, surtout dans nos régions où les berges sont en général plus ou moins fixées, mais il n'en reste pas moins vrai que la velléité de méandres est réelle.

\section{Considérations générales sur les travaux de confortation des ouvrages}

On conforte des ouvrages parce que des causes extérieures sont venues perturber l'équilibre initial de la rivière. Il est donc bien évident que si l'on supprime les causes, on stoppera un processus néfaste.
Par exemple toute diminution, ou à la limite suppression, des volumes dragués chaque année dans une rivière se traduira non seulement par un arrêt des processus d'érosion mais par une évolution favorable vers un nouvel équilibre et à la limite vers la situation initiale.

Autre exemple : toute diminution du débit d'une rivière par détournement : dans un bras secondaire, dans une zone de stockage, etc. se traduira par un exhaussement des fonds en aval de la zone de détournement. Ce sont peut-être là des cas d'école, souvent utopiques compte tenu de l'inertie à vaincre pour changer des situations existantes mais, il n'en reste pas moins qu'en premier lieu, il faut tenter d'agir sur les causes lorsqu'elles ne sont pas irréversibles. Une autorisation de dragage n'est jamais irréversible mais la réalisation d'un endiguement ou d'un recalibrage peut, par contre, difficilement être remise en cause.

Plaçons-nous dans le cas de causes irréversibles et supposons les situations suivantes:

\section{Evolution future menaçante}

Les fonds au droit de l'ouvrage ne sont pas encore suffisamment abaissés pour mettre l'ouvrage en danger, mais l'évolution de ces fonds, en aval ou en amont, fait craindre pour le futur une situation dangereuse.

Deux possibilités de protection peuvent s'envisager : une protection des fonds par revêtement (enrochements, bidim) ou par création d'un seuil en travers du lit pour maintenir ces fonds dans la situation présente, non encore dangereuse (fig. 21).

Dans ce cas, le revêtement ou le seuil doivent tenir compte de l'évolution future pour avoir quelques chances de rester stables.

Par exemple reprenons la figure 10 et soit à stabiliser la situation actuelle (repère 3 ) par un seuil $(S)$.

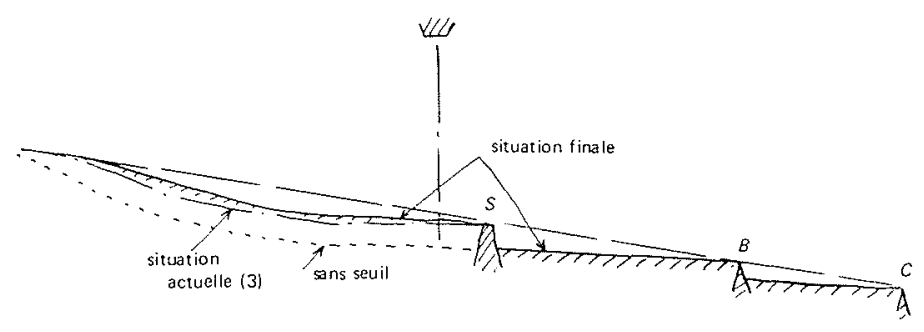

Figure 21

Ce seuil va bloquer les apports amont et contrarier l'évolution des fonds en amont. Par contre, en aval, la situation des fonds évoluera comme si le seuil n'existait pas. Il y aura donc, au pied de ce seuil, un affouillement qui peut être très important et entrainer sa ruine si ses fondations sont insuffisantes.

Bien entendu, la discontinuité des fonds au droit de ce seuil constitue une discontinuité dans les vitesses et les cotes d'eau, ce qui peut être rédhibitoire s'il est nécessaire de maintenir le passage d'un navigation.

Dans ce cas, on peut :

- soit répartir cette discontinuité en utilisant non pas un seuil mais un revêtement souple : enrochements, bidim... qui s'adaptera, plus ou moins bien, lorsque l'érosion régressive l'atteindra. 
- soit ne faire ni seuil ni revêtement, mais conforter tout de suite les culées et piles de l'ouvrage en vue de faire face à la situation d'équilibre finale, comme, il est indiqué dans le paragraphe suivant.

\section{Situation actuelle critique}

Les piles, culées... sont déjà déchaussées d'une façon très importante et nécessitent une confortation immédiate.

Il y a plusieurs solutions dont l'une consiste à réaliser un radier généralisé; c'est bien, lorsque les piles présentent encore suffisamment de fiche. Mais lorsque la fiche est insuffisante, il est nécessaire, localement, de réaliser soit un massif protégé (Fig. 22a) soit un rideau de palplanches encadrant la pile et qui injecté de béton constituera le renforcement recherché (Fig. 22b).

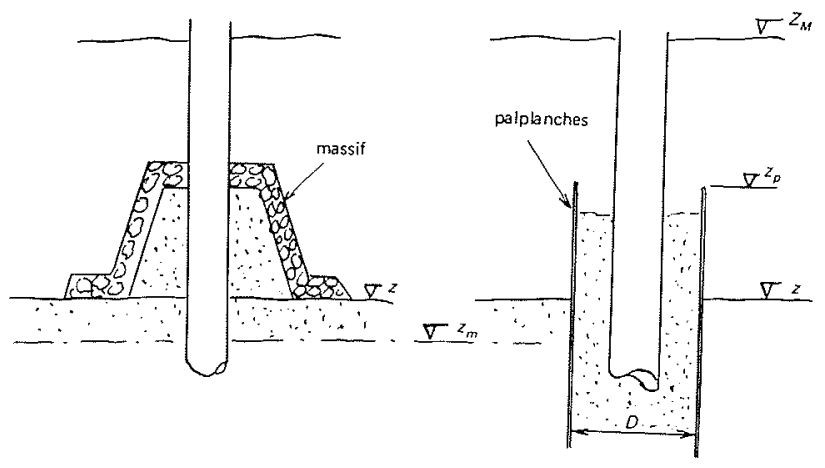

Figure 22
On ne saurait trop insister sur les deux points suivants :

1) l'encombrement du massif ou du rideau de palplanches doit être le plus faible possible de façon, d'une part, à réduire le moins possible la section de passage, d'autre part, à minimiser l'affouillement local qui, par exemple dans le cas du rideau de palplanches, reste égal à $2 D$ si la cote de crête de ces palplanches est largement au-dessus de la cote moyenne des fonds $z$; à cet égard $z_{p}$ a intérêt à être le plus proche possible de $z$ et même en-dessous si possible.

2) Il ne faut pas oublier qu'en cours de crue, la cote des fonds $z$ descend à la cote $z_{m}$ calculable par la formule (I) donnée précédemment et que, par exemple dans le cas du massif d'enrochements, il est souhaitable de prévoir à la base un tapis permettant de s'adapter à la configuration des fonds en cours de crue, si l'on veut que les talus du massif restent butés.

\section{Ouvrages intéressants à consulter}

NICOLLET - Hydraulique des ouvrages de franchissement des vallées fluviales. Rapport $L N H N^{\circ} E 43 / 80 / 20$.

RAMETTE - Cours d'eau sauvages, cours d'eau aménagés. Rapport LNH $N^{\circ}$ E40/79/10 bis.

RAMETTE - Guide d'hydraulique fluviale. Rapport $L N H$ HE 40/81/04.

Disponibles au Laboratoire National d'hydraulique, 6, Quai Watier, 78400 Chatou. 